Алгебра и анализ

Том. 17 (2005), вып. 5
St. Petersburg Math. J.

Vol. 17 (2006), No. 5, Pages 797-813

S 1061-0022(06)00930-7

Article electronically published on July 20, 2006

\title{
BIRKHOFF'S THEOREM FOR A FAMILY OF PROBABILITY SPACES
}

\author{
Y. SAFAROV
}

To the memory of O. A. Ladyzhenskaya

\begin{abstract}
Birkhoff's theorem on doubly stochastic matrices is extended to some countable families of discrete probability spaces with nonempty intersections.
\end{abstract}

A (possibly infinite) square matrix $\left\{w_{i j}\right\}_{i, j=1,2, \ldots}$ with nonnegative entries $w_{i j}$ is said to be doubly stochastic if its row and column sums are equal to one. The matrix $\left\{w_{i j}\right\}_{i, j=1,2, \ldots}$ can be identified with a function $w$ on the direct product of two discrete spaces $X=\left\{x_{1}, x_{2}, \ldots\right\}$ and $Y=\left\{y_{1}, y_{2}, \ldots\right\}$ such that $w\left(x_{i}, y_{j}\right)=w_{i j}, i, j=1,2, \ldots$. Under this identification, the matrix $\left\{w_{i j}\right\}_{i, j=1,2, \ldots}$ is doubly stochastic if and only if the restriction of $w$ to each subset $\left\{x_{i}\right\} \times Y$ or $X \times\left\{y_{j}\right\}$ is the density of a probability measure.

In accordance with Birkhoff's theorem Bi1,

(I) the extreme points of the convex set of doubly stochastic matrices are permutation matrices;

(II) the set of doubly stochastic matrices coincides with the closed convex hull of the set of permutation matrices.

Obviously, if (I) is true, then (II) follows from

$\left(\mathrm{II}^{\prime}\right)$ the set of doubly stochastic matrices coincides with the closed convex hull of the set of its extreme points.

Many proofs of Birkhoff's theorem are known for finite matrices (see, for example, $\mathrm{An}$, $\left[\overline{\mathrm{BR}}\right.$, or $[\mathbf{R O})$. The set of finite doubly stochastic matrices $\left\{w_{i j}\right\}_{i, j=1,2, \ldots, n}$ is compact. Therefore $\left(\mathrm{II}^{\prime}\right)$ is a particular case of the Krĕn-Milman theorem.

The problem of extending (I) and (II) to infinite matrices is known as Birkhoff's problem 111 [Bi2]. It was considered in [Is, RP], Ke], and [Sa]. In the infinite case, (I) remains true, but the validity of (II) depends on the choice of a topology. The set of doubly stochastic matrices is not compact in any natural locally convex topology on the linear space of infinite matrices (see $\S 3$ ). Therefore, the Kreln-Milman theorem is not applicable, and (II) must be proved separately.

Birkhoff's theorem has been generalized in many directions. We mention the following.

- In [Ho, $[\mathrm{Ka},[\mathrm{LLL},[\mathrm{ST}]$, and [Ti] the authors considered various subsets of the set of doubly stochastic matrices.

- The papers [CLMST, $\mathrm{Gr},[\mathrm{Le}$, and $\mathrm{Mu}$ dealt with classes of matrices with fixed, but not necessarily equal to one, row and column sums.

2000 Mathematics Subject Classification. Primary 05C50, 60C05.

Key words and phrases. Stochastic matrices, weighted graphs, Birkhoff's theorem.

(c)2006 American Mathematical Society 
- A measure $\mu$ on the direct product of two unit intervals is said to be doubly stochastic if $\mu(A \times X)=\mu(X \times A)=|A|$ for every Borel set $A \subseteq X$, where $|A|$ denotes the Lebesgue measure of $A$. The doubly stochastic measures were studied in [BS], [Do, $[\mathrm{Fe},[\mathrm{Li}],[\mathrm{KST}$, and $[\mathrm{Vi}$. In $\mathrm{Do}$ and $\mathrm{Li}$ the authors independently obtained a continuous analog of the first statement of Birkhoff's theorem.

- In [LMST] the authors considered nonnegative "hypermatrices", that is, nonnegative functions defined on the direct product of several discrete spaces.

Our aim in this paper is to obtain an analog of Birkhoff's theorem for a countable family of probability spaces with nonempty intersections. We consider a family $\Gamma$ of countable sets $\Omega_{1}, \Omega_{2}, \ldots$ and the convex set $\mathcal{S}(\Gamma)$ of nonnegative functions $w$ defined on the union $\Omega:=\bigcup_{k} \Omega_{k}$ and such that their restrictions $\left.w\right|_{\Omega_{k}}$ are the densities of probability measures on the sets $\Omega_{k}$. Each function in $\mathcal{S}(\Gamma)$ can be identified with a family of probability measures on the sets $\Omega_{k}$, coinciding on the intersections $\Omega_{i} \cap \Omega_{j}$.

Unlike in the papers mentioned above, we do not assume that the set $\Omega$ is a direct product and the sets $\Omega_{k}$ are its fibres. If $\Omega$ is infinite, we also consider convex subsets $\mathcal{S}(\Gamma, \mathcal{W}) \subset \mathcal{S}(\Gamma)$ that consist of functions satisfying certain decay conditions at infinity. Such conditions often appear in applications (see, e.g., Sa]).

The idea to consider several probability spaces with nonempty intersections is quite natural. This model seems to be a more adequate reflection of reality than the classical pattern with one probability space, where it is assumed implicitly that all events lying beyond the given scope either have probability zero or are totally unrelated to the space under consideration.

The sets $\mathcal{S}(\Gamma, \mathcal{W})$ themselves and their extreme points are determined by the layout of the sets $\Omega_{k}$. In $\S 2$ we join every two elements of $\Omega$ lying in the same set $\Omega_{k}$ by an edge and formulate our results in terms of the resulting graph $G$. Theorem 2.11 gives a complete description of the set of extreme points under the assumption that the multiplicity $\varkappa(g)$ of the covering $\left\{\Omega_{k}\right\}_{k=1,2, \ldots}$ does not exceed two for each $g \in \Omega$ (in other words, this means that each point $g \in \Omega$ belongs to at most two distinct sets $\left.\Omega_{k}\right)$. If $\varkappa(g) \leq 2$, then each function lying in the set of extreme points may only take the values $0, \frac{1}{2}$, 1 , and the subgraph associated with its support consists of isolated vertices and isolated odd primitive cycles (see Definition 2.1).

If $\Omega$ is the direct product of two discrete spaces $X$ and $Y$, and $\Gamma$ is the family of all sets of the form $\left\{x_{i}\right\} \times Y$ or $X \times\left\{y_{j}\right\}$, then $\varkappa \equiv 2$ and the corresponding graph $G$ has no odd primitive cycles. Therefore, (I) is a special case of Theorem 2.11. The graphs generated by multidimensional hypermatrices contain odd primitive cycles. Possibly, this explains the presence of the nonstandard extreme points discussed in [LMST]. Even primitive cycles were considered in $\mathrm{Gr},[\mathrm{Le}$, and $[\mathrm{Mu}$. Theorem 2.11 shows that, under the condition $\varkappa(g) \leq 2$, these cycles do not affect the structure of the set $\mathcal{S}(\Gamma)$.

The main result of $\S 3$ is Theorem 3.3 , where we prove $\left(\mathrm{II}^{\prime}\right)$ under certain conditions on the topology and the family $G$. These conditions include no assumptions about extreme points. Moreover, in $\S 3$ we do not use any results or definitions from $\S 2$. Therefore, that section can be read separately.

\section{§1. Notation AND DEFinitions}

1.1. Notation. We shall be using the following standard notation.

- $\mathbb{N}$ is the set of positive integers.

- $\left\{a_{1}, a_{2}, \ldots\right\}$ is the set with elements $a_{1}, a_{2}, \ldots$.

- \#A denotes the number of elements of a set $A$.

- $\operatorname{supp} w$ denotes the support of a function $w$. 
If $A$ is a subset of a real linear space $\mathcal{W}$, then

- ex $A$ is the set of extreme points of $A$;

- conv $A$ is the convex hull of $A$.

If $\mathcal{W}$ is equipped with a topology $\mathfrak{T}$, then

- $\overline{\operatorname{conv}} A$ is the closure of $\operatorname{conv} A$.

Let $\Gamma=\left\{\Omega_{1}, \Omega_{2}, \ldots\right\}$ be a family of countable sets $\Omega_{k}$, which may have nonempty intersection. Denoting $\Omega:=\bigcup_{k} \Omega_{k}$, we define

- $\mathcal{S}(\Gamma)$ to be the convex set of all nonnegative functions $w$ on $\Omega$ such that

$$
\sum_{g \in \Omega_{k}} w(g)=1 \quad \text { for all } \Omega_{k} \in \Gamma
$$

- $\mathcal{S}^{0}(\Gamma)$ to be the convex set of all nonnegative functions $w$ on $\Omega$ such that

$$
\sum_{g \in \Omega_{k}} w(g) \leq 1 \quad \text { for all } \Omega_{k} \in \Gamma
$$

- $\mathcal{P}(\Gamma)$ to be the set of functions $w \in \mathcal{S}(\Gamma)$ that only take the values 0 and 1 ;

- $\mathcal{P}^{0}(\Gamma)$ to be the set of functions $w \in \mathcal{S}^{0}(\Gamma)$ that take only the values 0 and 1.

If $w \in \mathcal{S}(\Gamma)$, then the restrictions $\left.w\right|_{\Omega_{k}}$ are the densities of probability measures on $\Omega_{k}$ such that

$$
\left.\mu_{i}\right|_{\Omega_{i} \cap \Omega_{j}}=\left.\mu_{j}\right|_{\Omega_{i} \cap \Omega_{j}}, \quad i, j=1,2, \ldots
$$

Conversely, every family of probability measures $\mu_{k}$ on $\Omega_{k}$ satisfying (1.1) generates a function $w \in \mathcal{S}(\Gamma)$.

If $\Omega_{k} \subseteq \Omega_{j}$, then all functions $w \in \mathcal{S}(\Gamma)$ are identically equal to zero on $\Omega_{j} \backslash \Omega_{k}$. In this case we can remove the set $\Omega_{j}$ from $\Gamma$ and all elements $g \in \Omega_{j} \backslash \Omega_{k}$ from $\Omega$ without changing the structure of the sets $\mathcal{S}(\Gamma)$ and $\mathcal{P}(\Gamma)$. Therefore, without loss of generality, we shall always assume that

$$
\Omega_{k} \backslash \Omega_{j} \neq \varnothing, \quad j \neq k .
$$

Given $g \in \Omega$ and a subset $\tilde{\Omega} \subseteq \Omega$, we define

- $\Gamma(g):=\left\{\Omega_{k} \in \Gamma: g \in \Omega_{k}\right\}, \Gamma(\tilde{\Omega}):=\bigcup_{g \in \tilde{\Omega}} \Gamma(g)$;

- $\varkappa(g):=\# \Gamma(g)$ and $\varkappa(\tilde{\Omega}):=\sup _{g \in \tilde{\Omega}} \Gamma(g)$.

Further on, we shall assume that

(a) $\# \Gamma(g)<\infty$ for all $g \in \Omega$.

We shall also need the following technical assumption:

$\left(\mathbf{a}_{1}\right)$ if $g \neq \tilde{g}$, then $\Gamma(g) \neq \Gamma(\tilde{g})$ for all $g, \tilde{g} \in \tilde{\Omega}$.

In the following two examples $\varkappa(g)=2$ for all $g \in \Omega$, and $\left(\mathbf{a}_{1}\right)$ is fulfilled for the entire set $\Omega=\bigcup_{k} \Omega_{k}$.

Example 1.1. Suppose that the family $\Gamma$ can be split into the union of two subfamilies $\Gamma^{+}=\left\{\Omega_{1}^{+}, \Omega_{2}^{+}, \ldots\right\}$ and $\Gamma^{-}=\left\{\Omega_{1}^{-}, \Omega_{2}^{-}, \ldots\right\}$ in such a way that $\Omega_{i}^{+} \cap \Omega_{j}^{+}=\varnothing, \Omega_{i}^{-} \cap \Omega_{j}^{-}=$ $\varnothing, \Omega=\bigcup_{i, j}\left(\Omega_{i}^{+} \cap \Omega_{j}^{-}\right)$and $\#\left(\Omega_{i}^{+} \cap \Omega_{j}^{-}\right)=1$ for all $i, j=1,2, \ldots$. Then $\Omega_{i}^{+}$and $\Omega_{j}^{-}$may be viewed as rows and columns of an $\left(m_{+} \times m_{-}\right)$-matrix, where $m^{ \pm}:=\# \Gamma^{ \pm}$. Under this identification, $\mathcal{S}(\Gamma)$ is the set of doubly stochastic matrices, and $\mathcal{P}(\Gamma)$ is the set of permutation matrices.

Example 1.2. Let $\Omega=\left\{g_{1}, g_{2}, \ldots, g_{n}\right\}, \Omega_{n}:=\left\{g_{n}, g_{1}\right\}$, and $\Omega_{k}:=\left\{g_{k}, g_{k+1}\right\}$ for $k=1,2, \ldots, n-1$. If $n$ is odd, then $\mathcal{P}(\Gamma)=\varnothing$, and $\mathcal{S}(\Gamma)$ consists of one function $w$ identically equal to $\frac{1}{2}$. 
1.2. The set $\mathcal{S}(\Gamma)$. It may well happen that $\mathcal{S}(\Gamma)=\varnothing$. Indeed,

$$
\# \Gamma=\sum_{g \in \Omega} \varkappa(g) w(g) \leq \varkappa(\Omega) \sum_{g \in \Omega} w(g), \quad w \in \mathcal{S}(\Gamma) .
$$

Therefore, $\mathcal{S}(\Gamma)=\varnothing$ for all finite nonsquare matrices (in which the number of rows is not equal to the number of columns). Relation (1.3) also implies the following more general statement.

Lemma 1.3. Assume that $\Omega$ coincides with the union of $m$ sets $\Omega_{k} \in \Gamma$. If $\# \Gamma>m \varkappa(\Omega)$, then $\mathcal{S}(\Gamma)=\varnothing$.

Proof. Under the conditions of the lemma, $\sum_{g \in \Omega} w(g) \leq m$ for all $w \in \mathcal{S}(\Gamma)$. If $\mathcal{S}(\Gamma) \neq$ $\varnothing$, then $\# \Gamma \leq m \varkappa(\Omega)$ by (1.3).

In particular, $\mathcal{S}(\Gamma)=\varnothing$ whenever $\# \Gamma=\infty, \varkappa(\Omega)<\infty$, and $\Omega$ coincides with the union of a finite collection of the sets $\Omega_{k} \in \Gamma$. The following lemma shows that this remains true under the less restrictive condition (a).

Lemma 1.4. Let $n \in \mathbb{N}$, and let $w$ be a nonnegative function on the union $G_{n}:=$ $\bigcup_{k \leq n} \Omega_{k}$ such that

$$
\sum_{g \in \Omega_{k}} w(g)=1 \text { for all } k \leq n \text { and } \sum_{g \in \Omega_{k} \cap G_{n}} w(g) \leq 1 \text { for all } k>n .
$$

If $\# \Gamma=\infty$ and condition (a) is fulfilled, then

$$
\sum_{g \in \Omega_{j} \cap G_{n}} w(g) \rightarrow 0, \quad j \rightarrow \infty .
$$

Proof. Since $\sum_{g \in G_{n}} w(g) \leq n<\infty$, for each $\varepsilon>0$ we can find a finite subset $G_{n, \varepsilon} \subseteq G_{n}$ such that

$$
0 \leq \sum_{g \in G_{n}} w(g)-\sum_{g \in G_{n, \varepsilon}} w(g)<\varepsilon .
$$

Condition (a) implies that $\sup \left\{j: \Omega_{j} \cap G_{n, \varepsilon}\right\}<\infty$. Therefore, the sum in (1.5) does not exceed $\varepsilon$ for all sufficiently large $j \in \mathbb{N}$.

Corollary 1.5. Assume that $\# \Gamma=\infty$ and condition (a) is fulfilled. If $\Omega$ coincides with the union of a finite collection of the sets $\Omega_{k} \in \Gamma$, then $\mathcal{S}(\Gamma)=\varnothing$.

1.3. The space $\mathcal{W}$. Let $\mathcal{W}$ be an arbitrary linear space of real-valued functions on $\Omega$ that includes $\mathcal{P}^{0}(\Gamma)$ and satisfies the following condition:

$(\mathbf{w})$ if $w \in \mathcal{W}$ and $|\tilde{w}| \leq|w|$, then $\tilde{w} \in \mathcal{W}$.

Since $\mathcal{P}^{0}(\Gamma) \subset \mathcal{W}$, the space $\mathcal{W}$ contains all functions with finite support. Since $\Omega$ is countable, $\mathcal{W}$ can be thought of as a subspace of the linear space of infinite real sequences (or a subspace of $\mathbb{R}^{m}$ if $\# \Omega=m<\infty$ ). Let

- $\mathcal{S}(\Gamma, \mathcal{W}):=\mathcal{S}(\Gamma) \cap \mathcal{W}$ and $\mathcal{S}^{0}(\Gamma, \mathcal{W}):=\mathcal{S}^{0}(\Gamma) \cap \mathcal{W}$.

Obviously, $\mathcal{P}(\Gamma) \subseteq \operatorname{ex} \mathcal{S}(\Gamma, \mathcal{W})$ and $\mathcal{P}^{0}(\Gamma) \subseteq \operatorname{ex} \mathcal{S}^{0}(\Gamma, \mathcal{W})$

If $\mathcal{W}$ contains the linear space

$$
\mathcal{W}_{1}:=\left\{w: \sup _{k} \sum_{g \in \Omega_{k}}|w(g)|<\infty\right\},
$$

then $\mathcal{S}(\Gamma, \mathcal{W})=\mathcal{S}(\Gamma)$ and $\mathcal{S}^{0}(\Gamma, \mathcal{W})=\mathcal{S}^{0}(\Gamma)$. In particular, this is the case if $\# \Omega<\infty$. If $\# \Omega=\infty$ and $\mathcal{W}_{1} \not \subset \mathcal{W}$, then the condition $w \in \mathcal{S}(\Gamma, \mathcal{W})$ imposes some restrictions on the behavior of the function $w \in \mathcal{S}(\Gamma)$ at infinity. 
Remark 1.6. In [Sa], I also considered the sets $\mathcal{S}\left(\Gamma, \Gamma_{1}\right)$ formed by the nonnegative functions $w$ such that

$$
\sum_{g \in \Omega_{k}} w(g) \leq 1 \text { for all } \Omega_{k} \in \Gamma \text { and } \sum_{g \in \Omega_{k}} w(g)=1 \text { for all } \Omega_{k} \in \Gamma_{1},
$$

where $\Gamma_{1}$ is a subset of $\Gamma$. Let $\Omega_{k}^{\prime}$ be the set obtained from $\Omega_{k}$ by adding one new element $g_{k}^{\prime}$ that does not belong to any other set $\Omega_{j}^{\prime}$. If $w \in \mathcal{S}\left(\Gamma, \Gamma_{1}\right)$ and

$$
w^{\prime}\left(g^{\prime}\right):= \begin{cases}w\left(g^{\prime}\right) & \text { for all } g^{\prime} \in \bigcup_{k} \Omega_{k}, \\ 1-\sum_{g \in \Omega_{k}} w(g) & \text { for } g^{\prime}=g_{k}^{\prime},\end{cases}
$$

then $w^{\prime} \in \mathcal{S}\left(\Gamma^{\prime}\right)$, where $\Gamma^{\prime}=\left\{\Omega_{1}^{\prime}, \Omega_{2}^{\prime}, \ldots\right\}$. By using this observation, the results of this paper can be extended to the sets $\mathcal{S}\left(\Gamma, \Gamma_{1}\right)$.

\section{§2. EXTREME POINTS}

2.1. The associated graph $G$. We join every two elements of $\Omega=\bigcup_{k} \Omega_{k}$ lying in the same set $\Omega_{k}$ by an edge and consider the resulting graph $G$. In this section we shall identify subsets of $\Omega$ with subgraphs of $G$, assuming that their vertices are adjacent if and only if they are adjacent in $G$ (in other words, the subgraphs include all edges joining their vertices). Then the sets $\Omega_{k}$ become complete subgraphs of the graph $G$. Note that $\Omega_{k}$ is not necessarily a maximal complete subgraph (a clique) and that there may be cliques in $G$ that do not contain any of the sets $\Omega_{k}$.

Recall that a path $\gamma$ in $G$ is a sequence $\left(g_{1}, g_{2}, \ldots, g_{m}\right)$ of vertices $g_{j}$ such that $\left(g_{j}, g_{j+1}\right)$ are distinct graph edges. A path $\gamma$ is said to be simple if each of its vertices is adjacent to at most two other vertices of $\gamma$. A path is called a cycle if $g_{m}=g_{1}$. A cycle is said to be odd (or even) if it contains an odd (or even) number of vertices.

Definition 2.1. We say that a simple path $\gamma \in G$ is primitive if none of the sets $\Omega_{k}$ contains more than two vertices of $\gamma$.

Note that the graph $G$ in Example 1.2 consists of one primitive cycle with $n$ vertices.

If a simple path $\gamma$ is not primitive, then

- either $\gamma$ has exactly three vertices lying in a subgraph $\Omega_{k}$,

- or $\gamma$ contains at least two nonconsecutive vertices that are adjacent in $G$.

If $\gamma=\left(g_{1}, g_{2}, \ldots, g_{m}\right)$ is a simple path in $G$ joining the vertices $g_{1}$ and $g_{m} \neq g_{1}$, then the shortest path of the form $\left(g_{1}, g_{j_{1}}, g_{j_{2}}, \ldots, g_{j_{l}}, g_{m}\right)$ is primitive. Therefore, any two vertices lying in a connected subgraph $G^{\prime}$ can be joined by a primitive path $\gamma \in G^{\prime}$. In particular, any path joining two given vertices and having the minimal possible number of vertices is primitive.

Lemma 2.2. Let $\gamma_{0}=\left(g_{1}, g_{2}, \ldots, g_{m}, g_{1}\right)$ be a simple cycle. Then there is a finite collection of cycles $\left\{\gamma_{1}^{\prime}, \gamma_{2}^{\prime}, \ldots \gamma_{n}^{\prime}\right\}$ such that:

(1) each cycle $\gamma_{i}^{\prime}$ either is primitive or has exactly three vertices lying in a subgraph $\Omega_{k}$;

(2) the set of vertices of the cycles $\gamma_{i}^{\prime}$ coincides with $\left\{g_{1}, g_{2}, \ldots, g_{m}\right\}$;

(3) $\gamma_{i}^{\prime}$ and $\gamma_{i+1}^{\prime}$ have exactly two common vertices and one common edge;

(4) if $j \geq 2$, then $\gamma_{i}^{\prime}$ and $\gamma_{i+j}^{\prime}$ have at most one common vertex.

Proof. Consider the shortest cycle $\gamma_{1}^{\prime}$ of the form

$$
\gamma_{1}^{\prime}=\left(g_{1}, g_{2}, \ldots, g_{j}, g_{l}, g_{l+1}, \ldots, g_{m}, g_{1}\right),
$$

which includes the edge $\left(g_{1}, g_{2}\right)$. This cycle either is primitive, or has three vertices lying in a subgraph $\Omega_{k}$. We denote by $\gamma_{1}$ the cycle $\left(g_{j}, g_{j+1}, \ldots, g_{l}, g_{j}\right)$ obtained from 
$\gamma_{0}$ by removing $\gamma_{1}^{\prime}$, and enumerate its vertices in such a way that $g_{l}=g_{1}$ and $g_{j}=$ $g_{2}$. Applying this procedure consecutively to $\gamma_{0}, \gamma_{1}, \gamma_{2}, \ldots$, after finitely many steps we obtain a collection of cycles $\gamma_{1}^{\prime}, \gamma_{2}^{\prime}, \ldots, \gamma_{n}^{\prime}$ satisfying conditions (1)-(4).

Lemma 2.3. Let $\tilde{G}$ be an arbitrary subgraph of $G$ satisfying condition $\left(\mathbf{a}_{1}\right)$. If $\varkappa(\tilde{G}) \leq 2$ and $\tilde{G}$ contains no primitive cycles, then every simple cycle $\gamma_{0} \in \tilde{G}$ is contained in a subgraph $\Omega_{k}$.

Proof. Let $\gamma_{1}^{\prime}, \gamma_{2}^{\prime}, \ldots, \gamma_{n}^{\prime}$ be the cycles constructed in Lemma 2.2. If $\tilde{G}$ contains no primitive cycles, then each cycle $\gamma_{i}^{\prime}$ lies in a subgraph $\Omega_{k_{i}}$. Now condition (3) of Lemma 2.2. condition $\left(\mathbf{a}_{1}\right)$, and the estimate $\varkappa(\tilde{G}) \leq 2$ imply that $\Omega_{k_{1}}=\Omega_{k_{2}}=\cdots=\Omega_{k_{n}}$.

Corollary 2.4. If a connected subgraph $G^{\prime}$ satisfies the conditions of Lemma 2.3, then every two of its vertices are joined by a unique primitive path.

Proof. Suppose that there are two distinct primitive paths in $G^{\prime}$ joining the same two vertices. Then $G^{\prime}$ contains a simple cycle $\gamma$ formed by edges and vertices of these paths. Since no three consecutive vertices of a primitive path belong to the same subgraph $\Omega_{k}$, the cycle $\gamma$ is not contained in any of these subgraphs. This contradicts Lemma 2.3.

In the general case, the absence of primitive cycles does not imply that every two vertices of a connected subgraph $G^{\prime}$ are joined by a unique primitive path.

Example 2.5. Let $\Omega=\left\{g_{0}, g_{1}, \ldots, g_{m+1}\right\}$ and $\Omega_{k}=\left\{g_{0}, g_{k}, g_{k+1}\right\}$, where $k=1, \ldots$, $m \geq 2$. Then $G$ contains no primitive cycles, but $g_{1}$ and $g_{m+1}$ are joined by the two distinct primitive paths $\left(g_{1}, g_{2}, \ldots, g_{m}, g_{m+1}\right)$ and $\left(g_{1}, g_{0}, g_{m+1}\right)$. In this example, $\varkappa\left(g_{0}\right)=m$ and $\varkappa(g) \leq 2$ for all other vertices $g$. If $m>2$, then $\varkappa(\Omega)>2$; if $m=2$, then $\left(\mathbf{a}_{1}\right)$ is not true because $\Gamma\left(g_{0}\right)=\Gamma\left(g_{2}\right)=\Gamma$.

Remark 2.6. Instead of $G$, the so-called intersection graph can be considered. The vertices of this graph are identified with the sets $\Omega_{k}$, and two vertices are adjacent if and only if the intersection of the corresponding sets is not empty. However, this construction seems to be less suitable for our purposes because the intersection graph may contain several distinct edges associated with the same element $g \in \Omega$.

2.2. Necessary conditions. Given $w \in \mathcal{W}$, we denote $G_{w}:=\operatorname{supp} w$ and

$$
\hat{w}(\tilde{G}):=\inf _{g \in \tilde{G}} \min \{w(g), 1-w(g)\}, \quad \tilde{G} \subseteq G_{w} .
$$

If $w \in \mathcal{S}(\Gamma)$, then

- $\hat{w}(\tilde{G}) \geq 0$ for all $\tilde{G} \subseteq G_{w}$; and

- $\hat{w}\left(G^{\prime}\right)>0$ for every finite connected subgraph $G^{\prime}$ containing more than one vertex.

The following two lemmas give necessary conditions on $G_{w}$, which are fulfilled for every extreme point $w$ of the set $\mathcal{S}(\Gamma, \mathcal{W})$.

Lemma 2.7. If $w \in \operatorname{ex} \mathcal{S}(\Gamma, \mathcal{W})$, then the graph $G_{w}$ contains no finite nonempty subgraphs $\tilde{G}$ satisfying the following two conditions:

(1) the intersection of $\tilde{G}$ with each subgraph $\Omega_{k}$ either is empty or contains exactly two distinct vertices;

(2) $\tilde{G}$ does not contain odd primitive cycles.

Proof. Let $w \in \mathcal{S}(\Gamma, \mathcal{W})$, and let $\tilde{G}$ be a finite subgraph of $G_{w}$ satisfying conditions (1) and (2). Consider an arbitrary connected component $G^{\prime}$ of the graph $\tilde{G}$. Since the subgraphs $\Omega_{k}$ are complete, the intersection $G^{\prime} \cap \Omega_{k}$ either is empty or coincides 
with $\tilde{G} \cap \Omega_{k}$ for each $\Omega_{k} \in \Gamma$. Therefore the subgraph $G^{\prime}$ also satisfies (1) and (2). In particular, from (1) it follows that $w(g) \in(0,1)$ for all $g \in G^{\prime}$. Since $G^{\prime}$ is finite, this implies that $\hat{w}\left(G^{\prime}\right)>0$.

By (1), every simple path in $G^{\prime}$ is primitive. By condition (2), it follows that the chromatic number of the graph $G^{\prime}$ equals two. In other words, the vertices of $G^{\prime}$ can be divided into two groups in such a way that no two vertices in one group are adjacent to each other. We denote $\varepsilon:=\hat{w}\left(G^{\prime}\right)$ and define functions $w_{\varepsilon}^{ \pm}$as follows:

- $w_{\varepsilon}^{ \pm}(g):=w(g)$ for all $g \notin G^{\prime}$;

- $w_{\varepsilon}^{ \pm}(g):=w(g) \pm \varepsilon$ if $g$ belongs to the first group of vertices;

- $w_{\varepsilon}^{ \pm}(g):=w(g) \mp \varepsilon$ if $g$ belongs to the second group of vertices.

Directly from the definition, it follows that $w_{\varepsilon}^{ \pm}(g) \in \mathcal{S}(\Gamma)$. Also, $w_{\varepsilon}^{ \pm} \in \mathcal{W}$ because $w \in \mathcal{W}$ and $\# \operatorname{supp}\left(w-w_{\varepsilon}^{ \pm}\right)<\infty$. Thus, $w_{\varepsilon}^{ \pm} \in \mathcal{S}(\Gamma, \mathcal{W})$ and $w=\frac{1}{2}\left(w_{\varepsilon}^{+}+w_{\varepsilon}^{-}\right) \notin \operatorname{ex} \mathcal{S}(\Gamma, \mathcal{W})$.

Corollary 2.8. If $w \in \operatorname{ex} \mathcal{S}(\Gamma, \mathcal{W})$, then each connected component of the graph $G_{w}$ satisfies condition $\left(\mathbf{a}_{1}\right)$.

Proof. If $g_{1}$ and $g_{2}$ lie in the same connected component of $G_{w}$ and $\Gamma\left(g_{1}\right)=\Gamma\left(g_{2}\right)$, then the subgraph $\tilde{G}:=\left\{g_{1}, g_{2}\right\}$ satisfies conditions (1) and (2) of Lemma 2.7.

Lemma 2.9. If $\varkappa(\Omega) \leq 2$ and $w \in \operatorname{ex} \mathcal{S}(\Gamma, \mathcal{W})$, then each connected component of the graph $G_{w}$ either consists of one vertex or contains a primitive cycle.

Proof. Let $w \in \mathcal{S}(\Gamma, \mathcal{W})$. Suppose that $G_{w}$ has a connected component $G^{\prime}$ that includes at least one edge and contains no primitive cycles. Then, by Corollaries 2.4 and 2.8 , every two vertices of $G^{\prime}$ are joined by a unique primitive path.

We fix $g_{0} \in G^{\prime}$ and denote by $\mathcal{G}_{n}$ the set of vertices in $G^{\prime}$ that are joined with $g_{0}$ by primitive paths with $n$ edges. Since the subgraphs $\Omega_{k}$ are complete, for every $k=1,2, \ldots$ there exists $n \geq 0$ such that $\Omega_{k} \subseteq \mathcal{G}_{n} \cup \mathcal{G}_{n+1}$. Moreover, if $\Omega_{k} \subseteq \mathcal{G}_{n} \cup \mathcal{G}_{n+1}$, then the intersection $\Omega_{k} \cap \mathcal{G}_{n}$ contains exactly one vertex, which we denote by $g_{k, n}$. Indeed, should $\Omega_{k} \cap \mathcal{G}_{n}$ contain another vertex $g_{k, n}^{\prime}$, then, joining $g_{0}$ with $g_{k, n}$ and $g_{k, n}^{\prime}$ by primitive paths and adding the edge $\left(g_{k, n}^{\prime}, g_{k, n}\right)$, we would obtain a simple cycle not lying in any of the sets $\Omega_{k}$. This contradicts Lemma 2.3 .

Since $\# G^{\prime}>1$, we have $w\left(g_{0}\right) \in(0,1)$. We denote

$$
\varepsilon_{0}:=\min \left\{\frac{1}{2}, \frac{1-w\left(g_{0}\right)}{2 w\left(g_{0}\right)}\right\}
$$

fix an arbitrary $\varepsilon \in\left(0, \varepsilon_{0}\right)$, and define

$$
\varepsilon_{k, 0}:=\varepsilon, \quad \varepsilon_{k, n+1}:=\varepsilon_{k, n} w\left(g_{k, n}\right)\left(1-w\left(g_{k, n}\right)\right)^{-1}, \quad n=1,2, \ldots .
$$

Consider the sequences of functions $w_{\varepsilon, n}^{+}$and $w_{\varepsilon, n}^{-}$defined as follows:

- $w_{\varepsilon, 0}^{ \pm}\left(g_{0}\right):=(1 \pm \varepsilon) w\left(g_{0}\right)$ and $w_{\varepsilon, 0}^{ \pm}(g):=w(g)$ for all $g \neq g_{0}$;

- $w_{\varepsilon, n+1}^{ \pm}(g):=w_{\varepsilon, n}^{ \pm}(g)$ for all $g \in \bigcup_{j \leq n} \mathcal{G}_{j}$;

- $w_{\varepsilon, n+1}^{ \pm}(g):=w(g)$ for all $g \notin \bigcup_{j \leq n+1} \mathcal{G}_{j}$;

- if $\Omega_{k} \subseteq \mathcal{G}_{n} \cup \mathcal{G}_{n+1}$, then

$$
w_{\varepsilon, n}^{ \pm}\left(g_{k, n}\right):=\left(1 \pm \varepsilon_{k, n}\right) w\left(g_{k, n}\right) \quad \text { and } \quad w_{\varepsilon, n+1}^{ \pm}(g):=\left(1 \mp \varepsilon_{k, n+1}\right) w(g)
$$

for all $g \in \Omega_{k} \cap \mathcal{G}_{n+1}$.

Obviously, $w(g)=\frac{1}{2}\left(w_{\varepsilon, n}^{+}(g)+w_{\varepsilon, n}^{-}(g)\right)$ for all $g \in \Omega$. Since $w \in \mathcal{S}(\Gamma)$, we have

$$
\frac{w\left(g_{k, n}\right)}{1-w\left(g_{k, n}\right)} \leq \frac{1-w(g)}{w(g)}, \quad g \in \Omega_{k} \cap \mathcal{G}_{n+1} .
$$


By using these inequalities, it is easy to prove that

$$
\varepsilon_{k, n} \leq \min \left\{\frac{1}{2}, \frac{1-w\left(g_{k, n}\right)}{2 w\left(g_{k, n}\right)}\right\}, \quad k=1,2, \ldots .
$$

Estimate (2.2) and the identity $w=\frac{1}{2}\left(w_{\varepsilon}^{+}+w_{\varepsilon}^{-}\right)$imply that

$$
0 \leq w_{\varepsilon, n}^{ \pm}(g) \leq w(g), \quad g \in G^{\prime}, \quad n=1,2, \ldots .
$$

If $\Omega_{k} \subseteq \mathcal{G}_{n} \cup \mathcal{G}_{n+1}$, then

$$
\sum_{g \in \Omega_{k}} w_{\varepsilon, n}^{ \pm}(g)=\left(1 \pm \varepsilon_{k, n}\right) w\left(g_{k, n}\right)+\left(1 \mp \varepsilon_{k, n+1}\right)\left(1-w\left(g_{k, n}\right)\right)=1 .
$$

Let $w_{\varepsilon}^{ \pm}(g):=\lim _{n \rightarrow \infty} w_{\varepsilon, n}^{ \pm}(g)$. Condition (w) and inequalities (2.3) imply that $w_{\varepsilon}^{ \pm} \in$ $\mathcal{W}$. By (2.4), we have $w_{\varepsilon}^{ \pm} \in \mathcal{S}(\Gamma)$. Therefore, $w_{\varepsilon}^{ \pm} \in \mathcal{S}(\Gamma, \mathcal{W})$ and $w=\frac{1}{2}\left(w_{\varepsilon}^{+}+w_{\varepsilon}^{-}\right)$is not an extreme point of the set $\mathcal{S}(\Gamma, \mathcal{W})$.

Remark 2.10. If $w \in \mathcal{P}(\Gamma)$, then $\#\left(\Omega_{k} \cap G_{w}\right)=1$ for all $\Omega_{k} \in \Gamma$, which implies that the subgraph $G_{w}$ has no edges. Conversely, every edgeless graph containing one element of each set $\Omega_{k}$ is the support of a function in $\mathcal{P}(\Gamma)$. If $w_{1}, w_{2} \in \mathcal{P}(\Gamma)$, then the subgraph $\tilde{G}:=\operatorname{supp}\left(w_{1}-w_{2}\right)$ satisfies conditions (1) and (2) of Lemma 2.7. The converse is not always true; a subgraph satisfying (1) and (2) may fail to coincide with the support of the difference $w_{1}-w_{2}$ with $w_{1}, w_{2} \in \mathcal{P}(\Gamma)$.

For example, suppose $\Omega=\left\{g_{1}, g_{2}, \ldots, g_{5}\right\}, \Omega_{1}=\left\{g_{2}, g_{3}\right\}, \Omega_{2}=\left\{g_{1}, g_{3}\right\}, \Omega_{3}=\left\{g_{1}, g_{2}\right\}$ and $\Omega_{4}=\left\{g_{4}, g_{5}\right\}$. Then the complete subgraph $\Omega_{4}$ satisfies conditions (1) and (2).

However, $\mathcal{S}(\Gamma)$ consists of functions $w$ such that $w\left(g_{1}\right)=w\left(g_{2}\right)=w\left(g_{3}\right)=\frac{1}{2}$ and $1-w\left(g_{5}\right)=w\left(g_{4}\right) \in[0,1]$. Therefore, $\mathcal{P}(\Gamma)=\varnothing$.

2.3. Necessary and sufficient conditions. The following theorem is the main result of this section.

Theorem 2.11. Assume that $\varkappa(\Omega) \leq 2$. Then $w \in \operatorname{ex} \mathcal{S}(\Gamma, \mathcal{W})$ if and only if $G_{w} \cap \Omega_{k} \neq$ $\varnothing$ for all $\Omega_{k} \in \Gamma$ and $w$ satisfies the following two conditions:

(1) each connected component of the graph $G_{w}$

$\left(1_{1}\right)$ either consists of one isolated vertex $g$,

$\left(1_{2}\right)$ or coincides with an odd primitive cycle $\gamma$;

(2) $w(g)=1$ in case $\left(1_{1}\right)$, and $\left.w\right|_{\gamma} \equiv \frac{1}{2}$ in case $\left(1_{2}\right)$.

Proof. If (1) is true and $G_{w} \cap \Omega_{k} \neq \varnothing$, then

$\left(1^{\prime}\right)$ for each $\Omega_{k} \in \Gamma$ the intersection $\Omega_{k} \cap G_{w}$ consists of

$\left(1_{1}^{\prime}\right)$ either one isolated vertex $g \in G_{w}$,

$\left(1_{2}^{\prime}\right)$ or two consecutive vertices of a primitive cycle $\gamma \in G_{w}$.

Indeed, since the graph $\Omega_{k}$ is complete, the intersection $\Omega_{k} \cap G_{w}$ lies in a connected component $G^{\prime}$ of the graph $G_{w}$. If $G^{\prime}$ is an isolated vertex, then $\left(1_{1}^{\prime}\right)$ is true. If $G^{\prime}$ coincides with a primitive cycle, then the intersection $\Omega_{k} \cap G_{w}$ cannot contain more than two vertices, because the cycle is primitive. On the other hand, $\Omega_{k} \cap G_{w}$ cannot consist of one vertex because $\varkappa(\Omega) \leq 2$. Therefore $\left(1_{2}^{\prime}\right)$ is fulfilled.

1. Let $G_{w} \cap \Omega_{k} \neq \varnothing$ for all $\Omega_{k} \in \Gamma$, and let conditions (1) and (2) be fulfilled.

The set of vertices of an odd primitive cycle can be represented as the union of three disjoint subsets such that $\Gamma(g) \cap \Gamma\left(g^{\prime}\right)=\varnothing$ for every pair of elements $g, g^{\prime}$ lying in the same subset. Therefore, $w$ coincides with $\frac{1}{2}\left(w_{1}+w_{2}+w_{3}\right)$, where $w_{i} \in \mathcal{P}^{0}(\Gamma) \subset \mathcal{W}$, $i=1,2,3$. This observation and conditions $\left(1^{\prime}\right),(2)$ imply that $w \in \mathcal{S}(\Gamma, \mathcal{W})$.

Assume that $w_{ \pm} \in \mathcal{S}(\Gamma, \mathcal{W})$ and $w=\frac{1}{2}\left(w_{+}+w_{-}\right)$. Then $\operatorname{supp} w_{ \pm} \subseteq G_{w}$ and $w_{ \pm}(g)=1$ at all isolated vertices $g \in G_{w}$. If $\gamma$ is a cycle in $G_{w}$, then $w_{ \pm}(g)+w_{ \pm}\left(g^{\prime}\right)=1$ for each 
pair of consecutive vertices $g, g^{\prime} \in \gamma$. Since $\gamma$ is odd, these relations imply that $w_{ \pm}(g)=\frac{1}{2}$ at all vertices $g \in \gamma$. Thus, $w_{ \pm}=w$ and, consequently, $w \in \operatorname{ex} \mathcal{S}(\Gamma, \mathcal{W})$.

2. Assume that $w \in \mathcal{S}(\Gamma, \mathcal{W})$ and (1) is fulfilled. Then $G_{w} \cap \Omega_{k} \neq \varnothing$ for all $\Omega_{k} \in \Gamma$. In case $\left(1_{1}^{\prime}\right)$ we have $w(g)=1$; in case $\left(1_{2}^{\prime}\right)$ we have $w(g)=\frac{1}{2}$ for all $g \in \gamma$, because the cycle $\gamma$ is odd. Thus, (2) follows from (1) and the fact that $w \in \mathcal{S}(\Gamma, \mathcal{W})$.

3. It remains to show that $(1)$ is true for all $w \in \operatorname{ex} \mathcal{S}(\Gamma, \mathcal{W})$. Assume that $w \in$ $\mathcal{S}(\Gamma, \mathcal{W})$ and consider an arbitrary connected component $G^{\prime}$ of $G_{w}$ containing more than one vertex. We are going to prove that $w \notin \operatorname{ex} \mathcal{S}(\Gamma, \mathcal{W})$ unless $G^{\prime}$ coincides with an odd primitive cycle. Corollary 2.8 and Lemma 2.9 allow us to assume without loss of generality that $G^{\prime}$ satisfies $\left(\mathbf{a}_{1}\right)$ and contains at least one primitive cycle.

4. If there is an even primitive cycle $\gamma \subset G^{\prime}$, then the estimate $\varkappa(\Omega) \leq 2$ implies that the subgraph $\tilde{G}=\gamma$ satisfies the conditions of Lemma 2.7 consequently, $w \notin \operatorname{ex} \mathcal{S}(\Gamma, \mathcal{W})$. Therefore, we assume that $G^{\prime}$ contains no even primitive cycles.

5. Let $\gamma=\left(g_{1}, g_{2}, \ldots, g_{l}, g_{1}\right)$ be an odd primitive cycle in $G^{\prime}$. Since $G_{w}$ satisfies $\left(\mathbf{a}_{1}\right)$, for each pair of consecutive vertices $g_{i}, g_{i+1} \in \gamma$ there exists a unique set $\Omega_{k_{i}}$ containing these vertices (we take $g_{l+1}:=g_{1}$ ). Denote by $G_{\gamma}$ the graph generated by the family of sets $\Gamma(\gamma)=\left\{\Omega_{k_{1}}, \ldots, \Omega_{k_{l}}\right\}$.

Let $G^{\prime} \backslash \gamma \neq \varnothing$. Since the graph $G^{\prime}$ is connected, the estimate $\varkappa(\Omega) \leq 2$ implies that at least one of the intersections $\Omega_{k_{i}} \cap G^{\prime}$ contains a vertex that does not belong to $\gamma$. Assume, for definiteness, that $\Omega_{k_{1}} \cap G^{\prime}$ contains a vertex $g_{0} \notin \gamma$.

5 (a). If $g_{0}$ belongs to $\Omega_{k_{j}}$ with $j>1$, then one of the primitive cycles $\left(g_{0}, g_{2}, \ldots, g_{j}, g_{0}\right)$ and $\left(g_{0}, g_{j+1}, \ldots, g_{l}, g_{1}, g_{0}\right)$ is even. If there exists a primitive path $\left(g_{0}, \tilde{g}_{1}, \ldots, \tilde{g}_{m}\right)$ in $G^{\prime}$ such that $\tilde{g}_{m} \in \Omega_{k_{j}}$ and $\tilde{g}_{i} \notin G_{\gamma}$ for all $i=1, \ldots, m-1$, then:

- if $j>1$, then either $\left(g_{0}, \tilde{g}_{1}, \ldots, \tilde{g}_{m}, g_{j+1}, g_{j+2}, \ldots, g_{l}, g_{1}, g_{0}\right)$ or $\left(g_{0}, \tilde{g}_{1}, \ldots, \tilde{g}_{m}\right.$, $\left.g_{j}, g_{j-1}, \ldots, g_{2}, g_{0}\right)$ is an even primitive cycle;

- if $j=1$, then one of the primitive cycles $\left(g_{0}, \tilde{g}_{1}, \ldots, \tilde{g}_{m}, g_{0}\right)$ and $\left(g_{0}, \tilde{g}_{1}, \ldots, \tilde{g}_{m}, g_{2}\right.$, $\left.\ldots, g_{l}, g_{1}, g_{0}\right)$ is even.

Therefore, the absence of even primitive cycles implies the following condition:

(5a) if the first edge of the path $\left(g_{0}, \tilde{g}_{1}, \ldots, \tilde{g}_{m}\right)$ is not contained in the subgraph $\Omega_{k_{1}}$, then none of its vertices $\tilde{g}_{i}$ belongs to $G_{\gamma}$.

Let $G^{\prime \prime}$ denote the subgraph of $G^{\prime}$ formed by the vertex $g_{0}$ and all the vertices $g$ joined with $g_{0}$ by such paths. By (5a), we have $G_{\gamma} \cap G^{\prime \prime}=\left\{g_{0}\right\}$. If $\Omega_{k} \notin \Gamma(\gamma)$ and $\Omega_{k} \cap G^{\prime \prime} \neq \varnothing$, then $\Omega_{k} \subseteq G^{\prime \prime}$ because the subgraph $\Omega_{k}$ is complete. Therefore, either $G^{\prime \prime}=\left\{g_{0}\right\}$ or $G^{\prime \prime}$ coincides with the graph generated by the family of sets $\Gamma^{\prime \prime}=\left\{\Omega_{n_{1}}, \Omega_{n_{2}}, \ldots\right\} \subset \Gamma$ such that $\Omega_{n_{i}} \notin \Gamma(\gamma)$ and $\Omega_{n_{i}} \cap G^{\prime \prime} \neq \varnothing$.

5(b). Assume that $G^{\prime \prime}=\left\{g_{0}\right\}$. Then $g_{0}$ belongs only to the set $\Omega_{k_{1}}$. Let

$$
\varepsilon_{1}:=\hat{w}\left(\left\{g_{0}, g_{1}, \ldots, g_{l}\right\}\right),
$$

and let $\varepsilon \in\left(0, \varepsilon_{1}\right)$. Consider the functions $w_{\varepsilon}^{+}$and $w_{\varepsilon}^{-}$defined as follows:

- $w_{\varepsilon}^{ \pm}(g)=w(g)$ for all $g \notin\left\{g_{0}, g_{1}, \ldots, g_{l}\right\}$;

- $w_{\varepsilon}^{ \pm}\left(g_{0}\right)=w\left(g_{0}\right) \pm \varepsilon$;

- $w_{\varepsilon}^{ \pm}\left(g_{1}\right)=w\left(g_{1}\right) \mp \varepsilon / 2$;

- $w_{\varepsilon}^{ \pm}\left(g_{i}\right)=w\left(g_{1}\right) \pm(-1)^{i-1} \varepsilon / 2$ for all $i=2, \ldots, l$.

Since $\varkappa(\Omega) \leq 2$, the vertex $g_{1}$ only belongs to the sets $\Omega_{k_{1}}$ and $\Omega_{k_{l}}$, and each vertex $g_{i}$ with $i \geq 2$ only belongs to the sets $\Omega_{k_{i-1}}$ and $\Omega_{k_{i}}$. Therefore, $w_{\varepsilon}^{ \pm} \in \mathcal{S}(\Gamma, \mathcal{W})$ and $w=\frac{1}{2}\left(w_{\varepsilon}^{+}+w_{\varepsilon}^{-}\right) \notin \operatorname{ex} \mathcal{S}(\Gamma, \mathcal{W})$.

$5(\mathrm{c})$. Assume that $G^{\prime \prime}$ has more than one vertex and contains no primitive cycles. Let $0<\varepsilon<\min \left\{\varepsilon_{0}, \varepsilon_{1}\right\}$, where $\varepsilon_{0}$ and $\varepsilon_{1}$ are as in (2.1) and (2.5). Consider the functions 
$w_{\varepsilon}^{+}$and $w_{\varepsilon}^{-}$defined as follows:

- if $g \notin \gamma$ and $g \notin G^{\prime \prime}$, then $w_{\varepsilon}^{ \pm}(g):=w(g)$;

- if $g \in \gamma$, then $w_{\varepsilon}^{ \pm}(g)$ is defined as in $5(\mathrm{~b})$;

- if $g \in G^{\prime \prime}$, then $w_{\varepsilon}^{ \pm}(g)$ is defined as in the proof of Lemma 2.9.

Since $G_{\gamma} \cap G^{\prime \prime}=\left\{g_{0}\right\}$, the inequalities $\varkappa(\Omega) \leq 2$ and (2.3) imply that $w_{\varepsilon}^{ \pm} \in \mathcal{S}(\Gamma, \mathcal{W})$. Thus $w=\frac{1}{2}\left(w_{\varepsilon}^{+}+w_{\varepsilon}^{-}\right) \notin \operatorname{ex} \mathcal{S}(\Gamma, \mathcal{W})$.

$5(\mathrm{~d})$. Finally, we assume that $G^{\prime \prime}$ contains an odd primitive cycle $\gamma^{\prime}$. By $(5 \mathrm{a}), g_{0}$ does not belong to $\gamma^{\prime}$. We consider the shortest primitive path $\gamma^{\prime \prime}=\left(g_{0}, g_{1}^{\prime \prime}, \ldots, g_{m}^{\prime \prime}, g^{\prime}\right)$ joining $g_{0}$ with $\gamma^{\prime}$ and enumerate the vertices of the cycle $\gamma^{\prime}=\left(g_{1}^{\prime}, g_{2}^{\prime}, \ldots, g_{n}^{\prime}, g_{1}^{\prime}\right)$ in such a way that $g_{1}^{\prime}=g^{\prime}$. Since $\varkappa(\Omega) \leq 2$, either the three vertices $g_{m}^{\prime \prime}, g_{n}^{\prime}, g_{1}^{\prime}$ or the three vertices $g_{m}^{\prime \prime}, g_{1}^{\prime}, g_{2}^{\prime}$ belong to the same set $\Omega_{k}$. For definiteness, assume the latter.

Let

$$
\varepsilon_{2}:=\hat{w}\left\{g_{0}, g_{1}, \ldots, g_{l}, g_{1}^{\prime}, \ldots, g_{n}^{\prime}, g_{1}^{\prime \prime}, \ldots, g_{m}^{\prime \prime}\right\}, \quad \varepsilon \in\left(0, \varepsilon_{2}\right),
$$

and let $w_{\varepsilon}^{ \pm}$be the functions defined as follows:

- if $g \notin \gamma \cup \gamma^{\prime} \cup \gamma^{\prime \prime}$, then $w_{\varepsilon}^{ \pm}(g):=w(g)$;

- if $g \in \gamma$ or $g=g_{0}$, then $w_{\varepsilon}^{ \pm}(g)$ is defined as in 6(b);

- $w_{\varepsilon}^{ \pm}\left(g_{i}^{\prime \prime}\right)=w\left(g_{i}^{\prime \prime}\right) \pm(-1)^{i} \varepsilon$ for all $i=1, \ldots, m$;

- $w_{\varepsilon}^{ \pm}\left(g_{1}^{\prime}\right)=w\left(g_{1}^{\prime}\right) \pm(-1)^{m+1} \varepsilon / 2$;

- $w_{\varepsilon}^{ \pm}\left(g_{i}^{\prime}\right)=w\left(g_{i}^{\prime}\right) \pm(-1)^{m+i-1} \varepsilon / 2$ for all $i=2, \ldots, n$.

Since $\varkappa(\Omega) \leq 2$, we have $w_{\varepsilon}^{ \pm} \in \mathcal{S}(\Gamma, \mathcal{W})$. Therefore, $w=\frac{1}{2}\left(w_{\varepsilon}^{+}+w_{\varepsilon}^{-}\right)$is not an extreme point of $\mathcal{S}(\Gamma, \mathcal{W})$.

Theorem 2.11 immediately implies the following statement.

Corollary 2.12. Let $\varkappa(\Omega) \leq 2$. Then

(1) $w(g) \in\left\{0, \frac{1}{2}, 1\right\}$ for all $w \in \operatorname{ex} \mathcal{S}(\Gamma, \mathcal{W})$ and $g \in \Omega$;

(2) ex $\mathcal{S}(\Gamma, \mathcal{W})=\mathcal{P}(\Gamma)$ whenever $G$ contains no odd primitive cycles.

Remark 2.13. If $\varkappa(\Omega) \leq 2$ and $G$ contains no odd primitive cycles, then $\Gamma$ can be split into the union of two disjoint subsets $\Gamma^{+}=\left\{\Omega_{1}^{+}, \Omega_{2}^{+}, \ldots\right\}$ and $\Gamma^{-}=\left\{\Omega_{1}^{-}, \Omega_{2}^{-}, \ldots\right\}$ in such a way that $\Omega_{i}^{+} \cap \Omega_{j}^{+}=\varnothing$ and $\Omega_{i}^{-} \cap \Omega_{j}^{-}=\varnothing$ for all $i, j=1,2, \ldots\left(\Omega_{i}\right.$ and $\Omega_{j}$ are included into the same set $\Gamma^{ \pm}$if every primitive path joining $\Omega_{i}$ and $\Omega_{j}$ has an odd number of edges). If, moreover, $\Omega=\bigcup_{i, j}\left(\Omega_{i}^{+} \cap \Omega_{j}^{-}\right)$and $\#\left(\Omega_{i}^{+} \cap \Omega_{j}^{-}\right)=1$ for all $i, j=1,2, \ldots$, then $\mathcal{S}(\Gamma)$ can be thought of as a set of doubly stochastic matrices (see Example 1.1).

\section{§3. Closed convex hull of extreme points}

3.1. Topologies on $\mathcal{W}^{\prime}$. Let $\mathcal{W}^{\prime}$ be the space of real-valued functions $w^{\prime}$ on $\Omega$ such that $\sum_{g \in \Omega}\left|w(g) w^{\prime}(g)\right|<\infty$ for all $w \in \mathcal{W}$. Further on we shall identify the functions $w^{\prime} \in \mathcal{W}^{\prime}$ with the corresponding linear functionals

$$
w \mapsto\left\langle w, w^{\prime}\right\rangle:=\sum_{g \in \Omega} w(g) w^{\prime}(g)
$$

on the space $\mathcal{W}$.

Let $\mathfrak{T}$ be a locally convex topology on $\mathcal{W}$ satisfying the following conditions:

$\left(\mathbf{w}_{1}\right)$ the topological dual $\mathcal{W}^{*}$ is a subspace of $\mathcal{W}^{\prime}$;

$\left(\mathbf{w}_{2}\right) \mathcal{W}^{*}$ contains all the functionals $w \mapsto \sum_{g \in \Omega_{k}} w(g), k=1,2, \ldots$

Condition $\left(\mathrm{w}_{2}\right)$ implies that $\overline{\operatorname{conv}} \operatorname{ex} \mathcal{S}(\Gamma, \mathcal{W}) \subseteq \mathcal{S}(\Gamma, \mathcal{W})$ and $\overline{\operatorname{conv}} \operatorname{ex} \mathcal{S}^{0}(\Gamma, \mathcal{W}) \subseteq \mathcal{S}^{0}(\Gamma, \mathcal{W})$

Let $\mathfrak{T}_{0}$ denote the topology of pointwise convergence on $\mathcal{W}$. If $\left(\mathbf{w}_{1}\right)$ is fulfilled and $\mathcal{W}^{*}$ consists of functions with finite support, then $\mathfrak{T}=\mathfrak{T}_{0}$. The Tikhonov theorem and 
Fatou lemma imply that the set $\mathcal{S}^{0}(\Gamma)$ is $\mathfrak{T}_{0}$-compact. Therefore, by the Kreı̆n-Milman theorem, $\mathcal{S}^{0}(\Gamma)$ coincides with the $\mathfrak{T}_{0}$-closure of the set conv ex $\mathcal{S}^{0}(\Gamma)$.

If $\# \Omega<\infty$, then $\operatorname{dim} \mathcal{W}<\infty, \mathfrak{T}=\mathfrak{T}_{0}$, the set $\mathcal{S}(\Gamma, \mathcal{W})$ is a compact convex polytope, and, consequently, $\mathcal{S}(\Gamma, \mathcal{W})=$ conv ex $\mathcal{S}(\Gamma, \mathcal{W})$. If at least one of the sets $\Omega_{k}$ is infinite, then it may well happen that the set $\mathcal{S}(\Gamma, \mathcal{W})$ is noncompact in any locally convex topology on $\mathcal{W}$. Indeed, if the linear functional $w \mapsto \sum_{g \in \Omega_{k}} w(g)$ is not continuous, then, as a rule, the set $\mathcal{S}(\Gamma, \mathcal{W})$ is not closed (there are exceptions, for instance, the case where $\mathcal{S}(\Gamma, \mathcal{W})=\varnothing$, but such exotic examples hardly deserve serious consideration). On the other hand, if the functional $w \mapsto \sum_{g \in \Omega_{k}} w(g)$ is continuous and $G_{k}=\left\{g_{1}, g_{2}, \ldots\right\}$, then $\mathcal{S}(\Gamma, \mathcal{W})$ may be compact only under the very restrictive assumption that

$$
\sup _{w \in \mathcal{S}(\Gamma, \mathcal{W})} \sum_{i>j}\left|w\left(g_{i}\right)\right| \underset{j \rightarrow \infty}{\rightarrow} 0
$$

(this follows from Theorem 1.2 in $[\mathrm{Sa}$, which is proved in the same way as Theorem 2.4 in Chapter 2 of $[\mathrm{Ru}]$ ).

In all examples known to the author, either $\overline{\operatorname{conv}} \operatorname{ex} \mathcal{S}(\Gamma, \mathcal{W})=\mathcal{S}(\Gamma, \mathcal{W})$, or at least one of the conditions $\left(\mathbf{w}_{1}\right)$ and $\left(\mathbf{w}_{2}\right)$ is not satisfied (see, e.g., [Is] or Remark 3.7 at the end of this section). It is quite possible that these conditions are sufficient. However, we can prove that conv $\operatorname{ex} \mathcal{S}(\Gamma, \mathcal{W})=\mathcal{S}(\Gamma, \mathcal{W})$ only under the following additional assumption:

$\left(\mathbf{a}_{2}\right)$ there exists $m \in \mathbb{N}$ such that $\left(\mathbf{a}_{21}\right)$ either $\Omega=\bigcup_{j=1}^{m} \Omega_{j}$,

$\left(\mathbf{a}_{22}\right)$ or none of the sets $\Omega_{k} \in\left\{\Omega_{m+1}, \Omega_{m+2}, \ldots\right\}$ lies in the union of a finite collection of other sets $\Omega_{j} \in \Gamma$.

In particular, condition $\left(\mathbf{a}_{2}\right)$ is satisfied if $\# \Gamma<\infty$, or if the number of finite sets $\Omega_{k}$ is finite and $\#\left(\Omega_{i} \cap \Omega_{j}\right)<\infty$ for all $i \neq j$.

3.2. An extension lemma. Put $G_{n}:=\bigcup_{k \leq n} \Omega_{k}$, and define

- $T_{0}$ to be the operator of extension by zero from $G_{n}$ to $\Omega$;

- $\mathcal{S}_{n}^{0}(\Gamma, \mathcal{W})$ to be the convex set of nonnegative functions $w$ on $G_{n}$ satisfying (1.4) and such that $T_{0} w \in \mathcal{W}$.

The role of condition $\left(\mathbf{a}_{22}\right)$ is clarified in the following lemma.

Lemma 3.1. If $\# \Gamma=\infty$ and conditions $(\mathbf{w}),(\mathbf{a})$, and $\left(\mathbf{a}_{22}\right)$ are fulfilled, then for each $n>m$ there exists a (nonlinear) extension operator $T_{n}$ from $G_{n}$ to $\Omega$ such that

(1) $T_{n}: \mathcal{S}_{n}^{0}(\Gamma, \mathcal{W}) \rightarrow \mathcal{S}(\Gamma, \mathcal{W})$

(2) $T_{n}: \operatorname{ex} \mathcal{S}_{n}^{0}(\Gamma, \mathcal{W}) \rightarrow \operatorname{ex} \mathcal{S}(\Gamma, \mathcal{W})$

(3) $\sup _{w \in \mathcal{S}_{n}^{0}(\Gamma, \mathcal{W})}\left\langle T_{0} w-T_{n} w, w^{\prime}\right\rangle \underset{n \rightarrow \infty}{\rightarrow} 0$ for all $w^{\prime} \in \mathcal{W}^{\prime}$

Proof. 1. Consider an arbitrary function $w \in \mathcal{S}_{n}^{0}(\Gamma, \mathcal{W})$. Let $\Gamma_{1}$ be the family of all sets $\Omega_{k} \in \Gamma$ such that $\sum_{g \in \Omega_{k} \cap G_{n}} w(g)=1$, let $\mathcal{G}_{1}$ be the union of these sets, and let $w_{1}$ be the extension of $w$ by zero to $\mathcal{G}_{1}$. By Lemma 1.4.

$$
\delta_{j}\left(w_{1}\right):=\sum_{g \in \Omega_{j} \cap \mathcal{G}_{1}} w_{1}(g) \underset{j \rightarrow \infty}{\longrightarrow} 0 .
$$

In particular, this implies that $\# \Gamma_{1}<\infty$.

Let $k_{1}:=\min \left\{k: \Omega_{k} \notin \Gamma_{1}\right\}$, let $\Gamma_{2}$ be the family of sets obtained from $\Gamma_{1}$ by adding the set $\Omega_{k_{1}}$, and let $\mathcal{G}_{2}$ be the union of the sets $\Omega_{k} \in \Gamma_{2}$. By $\left(\mathbf{a}_{22}\right)$, we have $\Omega_{k_{1}} \backslash G_{j} \neq \varnothing$ for all $j>k_{1}$. We choose an index $j$ such that $\delta_{i}\left(w_{1}\right)<\delta_{k_{1}}\left(w_{1}\right)$ for all $i>j$ and fix 
an arbitrary element $g_{1} \in \Omega_{k_{1}} \backslash G_{j}$. Let $w_{2}$ be the function on $\mathcal{G}_{2}$ defined as follows: $\left.w_{2}\right|_{\mathcal{G}_{1}}:=\left.w_{1}\right|_{\mathcal{G}_{1}}, w_{2}\left(g_{1}\right):=1-\delta_{k_{1}}\left(w_{1}\right)$, and $w_{2}\left(g^{\prime}\right):=0$ at all other vertices $g^{\prime}$. Then $\sum_{g \in \Omega_{k}} w_{2}(g)=1$ for all $\Omega_{k} \in \Gamma_{2}, \sum_{g \in \Omega_{k} \cap \mathcal{G}_{2}} w_{2}(g)<1$ for all $\Omega_{k} \in \Gamma \backslash \Gamma_{2}$, and

$$
\delta_{j}\left(w_{2}\right):=\sum_{g \in \Omega_{j} \cap \mathcal{G}_{2}} w_{2}(g) \underset{j \rightarrow \infty}{\longrightarrow} 0
$$

by Lemma 1.4 .

Let $k_{2}:=\min \left\{k: \Omega_{k} \notin \Gamma_{2}\right\}$, let $\Gamma_{3}:=\Gamma_{2} \cup\left\{\Omega_{k_{2}}\right\}$ and let $\mathcal{G}_{3}$ be the union of the sets $\Omega_{k} \in \Gamma_{3}$. We define $\Gamma_{2}^{\prime}:=\Gamma_{1} \cup \Gamma\left(g_{1}\right)$, choose $j \in \mathbb{N}$ so large that $\delta_{i}\left(w_{2}\right)<\delta_{k_{2}}\left(w_{2}\right)$ for all $i>j$, and fix an element $g_{2} \in \Omega_{k_{2}} \backslash G_{j}$ such that $\Gamma\left(g_{2}\right) \cap \Gamma_{2}^{\prime}=\varnothing$ (by (a) and (a $\left.\mathbf{a}_{22}\right)$, such an element does exist). We define a function $w_{3}$ on $\mathcal{G}_{3}$ by putting $\left.w_{3}\right|_{\mathcal{G}_{2}}:=\left.w_{2}\right|_{\mathcal{G}_{2}}$, $w_{3}\left(g_{2}\right):=1-\delta_{k_{2}}\left(w_{2}\right)$, and $w_{3}\left(g^{\prime}\right):=0$ at all other vertices $g^{\prime}$. Then

$$
\delta_{j}\left(w_{3}\right):=\sum_{g \in \Omega_{j} \cap \mathcal{G}_{3}} w_{3}(g) \underset{j \rightarrow \infty}{\longrightarrow} 0 .
$$

Now, we put $k_{3}:=\min \left\{k: \Omega_{k} \notin \Gamma_{3}\right\}$, and denote by $\mathcal{G}_{4}$ the union of all sets $\Omega_{k} \in$ $\Gamma_{4}:=\Gamma_{3} \cup\left\{\Omega_{k_{3}}\right\}$. We define $\Gamma_{3}^{\prime}:=\Gamma_{2}^{\prime} \cup \Gamma\left(g_{2}\right)$, choose $j \in \mathbb{N}$ such that $\delta_{i}\left(w_{3}\right)<\delta_{k_{3}}\left(w_{3}\right)$ for all $i>j$, fix an element $g_{3} \in \Omega_{k_{3}} \backslash G_{j}$ such that $\Gamma\left(g_{3}\right) \cap \Gamma_{3}^{\prime}=\varnothing$, and consider the function $w_{4}$ on $\mathcal{G}_{4}$ defined as follows: $\left.w_{4}\right|_{\mathcal{G}_{3}}=\left.w_{3}\right|_{\mathcal{G}_{3}}, w_{4}\left(g_{3}\right)=1-\delta_{k_{3}}\left(w_{3}\right)$, and $w_{4}\left(g^{\prime}\right)=0$ at all other vertices $g^{\prime}$.

Iterating this procedure, we obtain sequences of families $\Gamma_{j} \subset \Gamma$ of sets $\Omega_{k}$, their unions $\mathcal{G}_{j} \subset \Omega$, elements $g_{j} \in \mathcal{G}_{j+1} \backslash \mathcal{G}_{j}$, and functions $w_{j}$ on the sets $\mathcal{G}_{j}$ such that

(c1) $\Gamma_{j} \subset \Gamma_{j+1}$ and $\bigcup_{j=1}^{\infty} \Gamma_{j}=\Gamma$;

(c $\left.c_{2}\right)\left.w_{j+1}\right|_{\mathcal{G}_{j}}=\left.w_{j}\right|_{\mathcal{G}_{j}}$ and $\operatorname{supp}\left(w_{j+1}-w_{j}\right)=\left\{g_{j}\right\} \subset \Omega_{k_{j}}$;

$\left(c_{3}\right) \sum_{g \in \Omega_{k} \cap \mathcal{G}_{j}} w_{j}(g) \leq 1$ for all $\Omega_{k} \in \Gamma$ and $\sum_{g \in \Omega_{k}} w_{j}(g)=1$ for all $\Omega_{k} \in \Gamma_{j}$;

$\left(\mathrm{c}_{4}\right)$ for each $j \in \mathbb{N}$ there exists at most one index $i<j$ such that $\Gamma\left(g_{j}\right) \cap \Gamma\left(g_{i}\right) \neq \varnothing$.

Let $T_{n}$ be the operator defined by the relation $T_{n} w:=\lim _{j \rightarrow \infty} T_{0} w_{j}$, where the limit is taken in the topology $\mathfrak{T}_{0}$ of pointwise convergence. Since $0 \leq T_{n} w \leq 1$, properties $\left(\mathrm{c}_{1}\right)-\left(\mathrm{c}_{3}\right)$ imply that $T_{n} w \in \mathcal{S}(\Gamma)$.

Property $\left(\mathrm{c}_{2}\right)$ also implies

$\left(\mathrm{c}_{2}^{\prime}\right) \operatorname{supp}\left(T_{n} w-T_{0} w\right)=\gamma$, where $\gamma:=\left\{g_{1}, g_{2}, \ldots\right\}$.

We define subsets $\gamma^{\prime}$ and $\gamma^{\prime \prime}$ of $\gamma$ as follows:

- $g_{1} \in \gamma^{\prime}$ and $g_{j} \in \gamma^{\prime}$ whenever $\Gamma\left(g_{j}\right) \cap \Gamma\left(g_{i}\right)=\varnothing$ for all $i<j$;

- if $\Gamma\left(g_{j}\right) \cap \Gamma\left(g_{i}\right) \neq \varnothing$ for some $i<j$, then

- $g_{j} \in \gamma^{\prime}$ in the case where $g_{i} \in \gamma^{\prime \prime}$,

- $g_{j} \in \gamma^{\prime \prime}$ in the case where $g_{i} \in \gamma^{\prime}$.

By $\left(\mathrm{c}_{4}\right)$, we have $\gamma=\gamma^{\prime} \cup \gamma^{\prime \prime}, \gamma^{\prime} \cap \gamma^{\prime \prime}=\varnothing$, and $\Gamma\left(g^{\prime}\right) \cap \Gamma\left(g^{\prime \prime}\right)=\varnothing$ for every pair of distinct elements $g^{\prime}, g^{\prime \prime} \in \gamma^{\prime}$ and every pair of distinct elements $g^{\prime}, g^{\prime \prime} \in \gamma^{\prime \prime}$. Therefore, the characteristic functions $\chi^{\prime}$ and $\chi^{\prime \prime}$ of the sets $\gamma^{\prime}$ and $\gamma^{\prime \prime}$ belong to $\mathcal{P}^{0}(\Gamma)$. Since $T_{n} w-T_{0} w \leq 1$, we have $T_{n} w-T_{0} w \leq \chi^{\prime}+\chi^{\prime \prime}$. This estimate and condition (w) imply that $T_{n} w \in \mathcal{W}$. Thus, $T_{n} w \in \mathcal{S}(\Gamma, \mathcal{W})$, that is, $T_{n}$ satisfies (1).

2. Suppose that $w \in \operatorname{ex} \mathcal{S}_{n}^{0}(\Gamma, \mathcal{W})$ and $T_{n} w \notin \operatorname{ex} \mathcal{S}(\Gamma, \mathcal{W})$. Then there exists a function $\tilde{w} \not \equiv 0$ on $\Omega$ such that $T_{n} w \pm \tilde{w} \in \mathcal{S}(\Gamma, \mathcal{W})$. Property $\left(\mathrm{c}_{2}^{\prime}\right)$ and the membership relations $T_{n} w \in \mathcal{S}(\Gamma)$ and $T_{n} w \pm \tilde{w} \in \mathcal{S}(\Gamma)$ imply that

$$
\operatorname{supp} \tilde{w} \subseteq \operatorname{supp} T_{n} w \subseteq G_{n} \cup\left\{g_{1}, g_{2}, \ldots\right\},
$$

and $\sum_{g \in \Omega_{j} \cap G_{n}}|\tilde{w}(g)| \leq 1$ for all $\Omega_{k} \in \Gamma$. 
Let $w_{\varepsilon}^{ \pm}$denote the restrictions of the nonnegative functions $T_{n} w \pm \varepsilon \tilde{w}$ to the set $G_{n}$. By Lemma 1.4.

$$
\delta:=\sup _{\Omega_{j} \notin \Gamma_{1}} \sum_{g \in \Omega_{j} \cap \mathcal{G}_{1}} w(g)<1 .
$$

Therefore, $w_{\varepsilon}^{ \pm} \in \mathcal{S}_{n}^{0}(\Gamma, \mathcal{W})$ for all $\varepsilon<1-\delta$. Since $w_{\varepsilon}^{+}+w_{\varepsilon}^{-}=w$ and $w \in \operatorname{ex} \mathcal{S}_{n}^{0}(\Gamma, \mathcal{W})$, it follows that $\left.\tilde{w}\right|_{\mathcal{G}_{1}} \equiv 0$. Now, using $\left(\mathrm{c}_{2}\right)$, the identities

$$
w_{j+1}\left(g_{j}\right)=1-\delta_{k_{j}}\left(w_{j}\right)=1-\sum_{g \in \Omega_{k_{j}} \backslash\left\{g_{j}\right\}} w_{j}(g),
$$

and induction on $j$, we see that $\tilde{w}\left(g_{j}\right)=0$ for all $g_{j}$. Thus, $\tilde{w} \equiv 0$. This contradiction proves (2).

3. Let $w^{\prime} \in \mathcal{W}^{\prime}$. Suppose that $\sup _{w \in \mathcal{S}_{n}^{0}(\Gamma, \mathcal{W})}\left\langle T_{0} w-T_{n} w, w^{\prime}\right\rangle$ does not converge to zero. Then there exists $\delta>0$ and a sequence of functions $w_{n} \in \mathcal{S}_{n}^{0}(\Gamma, \mathcal{W})$ such that $\left\langle T_{0} w_{n}-T_{n} w_{n}, w^{\prime}\right\rangle \geq \delta$. If $\tilde{w}_{n}:=T_{n} w_{n}-T_{0} w_{n}$, then $\tilde{w}_{n} \in \mathcal{S}^{0}(\Gamma, \mathcal{W}), \operatorname{supp} \tilde{w}_{n}=$ $\left\{g_{1}^{n}, g_{2}^{n}, \ldots\right\} \subset \Omega \backslash G_{n}$, and

$$
\sum_{j=1}^{\infty}\left|\tilde{w}_{n}\left(g_{j}^{n}\right) w^{\prime}\left(g_{j}^{n}\right)\right| \geq\left\langle T_{n} w_{n}-T_{0} w_{n}, w^{\prime}\right\rangle \geq \delta,
$$

where the sets $\left\{g_{1}^{n}, g_{2}^{n}, \ldots\right\}$ are the sets of vertices associated with the functions $w_{n}$ (see the first part of the proof).

We consider arbitrary finite subsets $H_{n} \subset\left\{g_{1}^{n}, g_{2}^{n}, \ldots\right\}$ such that

$$
\sum_{g \in H_{n}}\left|\tilde{w}_{n}(g) w^{\prime}(g)\right| \geq \delta / 2 .
$$

Since $H_{n} \cap G_{n}=\varnothing$, (a) implies that $\Gamma\left(H_{n}\right) \cap \Gamma\left(H_{n+j}\right)=\varnothing$ for all sufficiently large $j \in \mathbb{N}$. Therefore, we can choose a subsequence $\left\{H_{n_{i}}\right\}_{i=1,2, \ldots}$ of the sequence $\left\{H_{n}\right\}_{n=1,2, \ldots}$ in such a way that

$$
\Gamma\left(H_{n_{i}}\right) \cap \Gamma\left(H_{n_{j}}\right)=\varnothing, \quad n_{i} \neq n_{j} .
$$

Let $\tilde{w}(g):=0$ for all $g \notin \bigcup_{i=1}^{\infty} H_{n_{i}}$, and let $\tilde{w}(g):=w_{n_{i}}(g)$ for all $g \in H_{n_{i}}$. In the first part of the proof it was shown that the function $\tilde{w}$ is estimated on every set $H_{n_{i}}$ by the sum of two functions in $\mathcal{P}^{0}(\Gamma)$. By (3.1), it follows that $\tilde{w}$ is estimated by the sum of two functions in $\mathcal{P}^{0}(\Gamma)$ on the entire set $\Omega$. Therefore, $\tilde{w} \in \mathcal{W}$. On the other hand, we have $\sum_{g \in \Omega}\left|\tilde{w}(g) w^{\prime}(g)\right|=\infty$, which contradicts the condition $w^{\prime} \in \mathcal{W}^{\prime}$. This proves (3).

Remark 3.2. From our definition of the operator $T_{n}$ it is clear that $T_{n} w \in \mathcal{P}(\Gamma)$ whenever $w$ only takes the values 0 and 1 . This observation can be used for constructing functions $w \in \mathcal{P}(\Gamma)$.

3.3. Closed convex hull. Lemma 3.1 allows us to prove the following statement.

Theorem 3.3. If conditions $(\mathbf{a}),(\mathbf{w})$, and $\left(\mathbf{a}_{2}\right)$ are fulfilled, then

$$
\mathcal{S}(\Gamma, \mathcal{W})=\overline{\operatorname{conv}} \operatorname{ex} \mathcal{S}(\Gamma, \mathcal{W})
$$

in any topology $\mathfrak{T}$ satisfying $\left(\mathbf{w}_{1}\right)$ and $\left(\mathbf{w}_{2}\right)$.

Proof. We fix an arbitrary function $w \in \mathcal{S}(\Gamma, \mathcal{W})$. In view of $\left(\mathbf{w}_{2}\right)$, it suffices to prove that $w \in \overline{\text { conv }} \operatorname{ex} \mathcal{S}(\Gamma, \mathcal{W})$. Recall that, by the separation theorem, $\overline{\operatorname{conv}} \operatorname{ex} \mathcal{S}(\Gamma, \mathcal{W})$ coincides with the weak closure of the convex set conv ex $\mathcal{S}(\Gamma, \mathcal{W})$. 

Let

1. Assume that there exist $g_{1}, g_{2}, \ldots \in \operatorname{supp} w$ such that $\Gamma\left(g_{1}\right)=\Gamma\left(g_{j}\right)$ for all $j \geq 2$.

- $w_{i}\left(g_{i}\right):=\sum_{j \geq 1} w\left(g_{j}\right)$;

- $w_{i}\left(g_{j}\right):=0$ for all $j \neq i$;

- $w_{i}(g)=w(g)$ for all $g \notin\left\{g_{1}, g_{2}, \ldots\right\}$.

Then $w_{i} \in \mathcal{S}(\Gamma, \mathcal{W})$ and, by $\left(\mathbf{w}_{1}\right)$, there exists a sequence of finite convex linear combinations of the functions $w_{i}$ that is weakly convergent to $w$. Since the set of all finite intersections of the sets $\Omega_{k}$ is countable, this implies that $w$ is contained in the weak sequential closure of the set of all functions $\tilde{w} \in \mathcal{S}(\Gamma, \mathcal{W})$ whose supports satisfy condition $\left(\mathbf{a}_{1}\right)$. Therefore, without loss of generality, we may assume that supp $w$ satisfies $\left(\mathbf{a}_{1}\right)$.

2. If $\# \Gamma<\infty$, then $\# \operatorname{supp} w<\infty$ by $\left(\mathbf{a}_{1}\right)$. In this case $w$ belongs to conv ex $\mathcal{S}(\Gamma, \mathcal{W})$. If $\# \Gamma=\infty$ and $\left(\mathbf{a}_{21}\right)$ is true, then $\mathcal{S}(\Gamma)=\overline{\operatorname{conv}} \operatorname{ex} \mathcal{S}(\Gamma)=\varnothing$ (see Corollary 1.5). In what follows it is assumed that $\# \Gamma=\infty$ and $\Gamma$ satisfies $\left(\mathbf{a}_{22}\right)$.

3. Assume that $w_{n}^{\star} \in \mathcal{S}_{n}(\Gamma)$ and $\operatorname{supp} w_{n}^{\star}$ satisfies $\left(\mathbf{a}_{1}\right)$. Suppose that $\Omega_{k} \cap \operatorname{supp} w_{n}^{\star}=$ $\left\{g_{1}^{k}, g_{2}^{k}, \ldots\right\}$ and $\Gamma_{i, k}^{(n)}:=\Gamma\left(g_{i}^{k}\right) \cap\left\{\Omega_{n+1}, \Omega_{n+2}, \ldots\right\}$, where $k=1,2, \ldots, n$. From $\left(\mathbf{a}_{1}\right)$, (a) and (1.5) it follows that

$$
\sup _{\Omega_{j} \in \Gamma_{i, k}^{(n)}} \sum_{g \in \Omega_{j} \cap G_{n}} w_{n}^{\star}(g) \underset{i \rightarrow \infty}{\longrightarrow} 0
$$

for all $k=1,2, \ldots, n$. Also, if $\# \Omega_{k}=\infty$, then

$$
v_{n}\left(g_{i}^{k}\right):=\sum_{j>i} w_{n}^{\star}\left(g_{j}^{k}\right) \underset{i \rightarrow \infty}{\longrightarrow} 0,
$$

and $\Gamma\left(g_{i}^{k}\right) \cap\left\{\Omega_{1}, \Omega_{2}, \ldots, \Omega_{n}\right\}=\left\{\Omega_{k}\right\}$ whenever $i$ is sufficiently large. Therefore, for all sufficiently large $i \in \mathbb{N}$ the functions $w_{n, i}^{\star}$ defined by the formulas

- $w_{n, i}^{\star}\left(g_{j}^{k}\right):=w_{n}^{\star}\left(g_{j}^{k}\right)$ for all $j=1, \ldots, i-1$,

- $w_{n, i}^{\star}\left(g_{i}^{k}\right):=v_{n}\left(g_{i}^{k}\right)$, and

- $w_{n, i}^{\star}\left(g_{j}^{k}\right)=0$ for all $j>i$

belong to $\mathcal{S}_{n}(\Gamma)$. Each of these functions has compact support and, therefore, coincides with a finite convex linear combination of functions $w_{n, i}^{j}$ belonging to conv ex $\mathcal{S}_{n}(\Gamma)$. By $\left(\mathbf{w}_{1}\right)$, the sequence $\left\{T_{0} w_{n, i}^{\star}\right\}_{i=1,2, \ldots}$ weakly converges to $T_{0} w_{n}^{\star}$. Thus, $T_{0} w_{n}^{\star}$ is contained in the weak sequential closure of the set $T_{0}\left(\operatorname{conv} \operatorname{ex} \mathcal{S}_{n}(\Gamma)\right)$.

4. Let $w_{n}^{\star}:=\left.w\right|_{G_{n}}$. By $\left(\mathbf{w}_{1}\right)$, we have $\left\langle w-T_{0} w_{n}^{\star}, w^{\prime}\right\rangle \underset{n \rightarrow \infty}{\rightarrow} 0$ for all $w^{\prime} \in \mathcal{W}^{\prime}$.

Since $\operatorname{supp} w$ satisfies $\left(\mathbf{a}_{1}\right)$, the same is true for $\operatorname{supp} w_{n}^{\star}$. Let $w_{n, i}^{\star}:=\sum_{j} \alpha_{j} w_{n, i}^{j}$ be the finite convex linear combination of functions $w_{n, i}^{j} \in \operatorname{ex} \mathcal{S}_{n}(\Gamma)$ introduced in the preceding part of the proof. Then $\left\langle T_{0} w_{n}^{\star}-T_{0} w_{n, i}, w^{\prime}\right\rangle \underset{i \rightarrow \infty}{\rightarrow} 0$ for each function $w^{\prime} \in \mathcal{W}^{\prime}$ and for each $n \in \mathbb{N}$.

Define $w_{n, i}:=\sum_{j} \alpha_{j} T_{n} w_{n, i}^{j}$. Conditions (2) and (3) of Lemma 3.1 imply that $w_{n, i} \in$ $\operatorname{conv} \operatorname{ex} \mathcal{S}(\Gamma, \mathcal{W})$ and

$$
\begin{aligned}
& \left\langle T_{0} w_{n, i}^{\star}-w_{n, i}, w^{\prime}\right\rangle=\sum_{j} \alpha_{j}\left\langle T_{0} w_{n, i}^{j}-T_{n} w_{n, i}^{j}, w^{\prime}\right\rangle \\
& \quad \leq \sup _{w \in \mathcal{S}_{n}^{0}(\Gamma, \mathcal{W})}\left\langle T_{0} w-T_{n} w, w^{\prime}\right\rangle \underset{n \rightarrow \infty}{\rightarrow} 0, \quad w^{\prime} \in \mathcal{W}^{\prime} .
\end{aligned}
$$

From the above, it follows that, for each function $w^{\prime} \in \mathcal{W}^{\prime}$, choosing a sufficiently large $n$ and then a sufficiently large $i$, we can make the sum on the right-hand side of 
the inequality

$$
\begin{gathered}
\left|\left\langle w-w_{n, i}, w^{\prime}\right\rangle\right| \leq\left|\left\langle w-T_{0} w_{n}^{\star}, w^{\prime}\right\rangle\right|+\left|\left\langle T_{0} w_{n}^{\star}-T_{0} w_{n, i}^{\star}, w^{\prime}\right\rangle\right|+\left|\left\langle T_{0} w_{n, i}^{\star}-w_{n, i}, w^{\prime}\right\rangle\right| \\
\leq\left|\left\langle w-T_{0} w_{n}^{\star}, w^{\prime}\right\rangle\right|+\left|\left\langle T_{0} w_{n}^{\star}-T_{0} w_{n, i}^{\star}, w^{\prime}\right\rangle\right|+\sup _{w \in \mathcal{S}_{n}^{0}(\Gamma, \mathcal{W})}\left\langle T_{0} w-T_{n} w, w^{\prime}\right\rangle
\end{gathered}
$$

arbitrarily small. By the separation theorem, this implies (3.2).

Remark 3.4. If we enlarge the dual space $\mathcal{W}^{*}$, then the topology on $\mathcal{W}$ becomes finer. Therefore, without loss of generality, in Theorem 3.3 we can replace $\left(\mathbf{w}_{1}\right)$ with the stronger condition

$$
\left(\mathrm{w}_{1}^{\prime}\right) \mathcal{W}^{*}=\mathcal{W}^{\prime}
$$

It can also be assumed that $\mathfrak{T}$ is the strongest topology satisfying $\left(\mathbf{w}_{1}^{\prime}\right)$ (this topology is called the Mackey topology). Finally, since $\mathcal{S}(\Gamma) \subset \mathcal{W}_{1}$, we can always assume that $\mathcal{W} \subseteq \mathcal{W}_{1}$ because reduction of $\mathcal{W}$ enlarges the space $\mathcal{W}^{\prime}$. If we take a smaller space $\mathcal{W} \subset \mathcal{W}_{1}$ (for example, one may wish to consider functions whose restrictions to $\Omega_{k}$ belong to a weighted space $l^{p}$ ), then Theorem 3.3 gives a stronger result which is valid for a narrower class of functions $w \in \mathcal{S}(\Gamma, \mathcal{W})$.

Remark 3.5. The topological space $(\mathcal{W}, \mathfrak{T})$ may be incomplete. However, if $\mathcal{W}=\mathcal{W}^{\prime \prime}$ and $\mathcal{W}^{*}=\mathcal{W}^{\prime}$, then $\mathcal{W}$ is complete in the Mackey topology and is weakly sequentially complete (see, e.g., Subsection 30.5 in $[\mathrm{K}]$ ).

Example 3.6. Let us consider the coarsest topology on $\mathcal{W}=\mathcal{W}_{1}$ for which $\left(\mathbf{w}_{1}\right)$ and $\left(\mathbf{w}_{2}\right)$ are satisfied and with respect to which all the functionals $w \mapsto w(g)$ are continuous. This topology is generated by the seminorms $p_{k}(w):=\sum_{g \in \Omega_{k}}|w(g)|$ and $p_{g}(w):=|w(g)|$; consequently, it is metrizable. Therefore, Theorem 3.3 implies that, under conditions (a) and $\left(\mathbf{a}_{2}\right)$, for every function $w \in \mathcal{S}(\Gamma)$ there exists a sequence of functions $w_{n} \in$ conv ex $\mathcal{S}(\Gamma)$ such that

$$
\left|w(g)-w_{n}(g)\right| \underset{n \rightarrow \infty}{\rightarrow} 0 \text { and } \sum_{g \in \Omega_{j}}\left|w(g)-w_{n}(g)\right| \underset{n \rightarrow \infty}{\rightarrow} 0
$$

for all $g \in \Omega$ and $k=1,2, \ldots$. In $[\mathrm{Ke}$, (3.2) was proved for this coarsest topology on the space of infinite matrices.

A topology $\mathfrak{T}$ satisfying conditions $\left(\mathbf{w}_{1}\right)$ and $\left(\mathbf{w}_{2}\right)$ (in particular, the Mackey topology), may well be nonmetrizable. Therefore, generally, (3.2) does not imply the existence of a sequence of convex linear combinations $w_{n} \in \operatorname{conv}$ ex $\mathcal{S}(\Gamma, \mathcal{W})$ convergent to a given function $w \in \mathcal{S}(\Gamma, \mathcal{W})$. Possibly, Theorem 3.3 can be improved in this direction (note that in parts 1 and 3 of the proof we spoke about sequential closures).

Remark 3.7. It seems to be natural to consider the closure of conv ex $\mathcal{S}(\Gamma)$ with respect to the norm $\|w\|_{\mathcal{S}}:=\sup _{j} \sum_{g \in \Omega_{j}}|w(g)|$ on the space $\mathcal{W}_{1}$. However, this closure does not always coincide with $\mathcal{S}(\Gamma)$.

Indeed, let $\Gamma$ be an infinite family of mutually disjoint sets $\Omega_{k}$, and let $\# \Omega_{k}=k$. Then $\operatorname{ex} \mathcal{S}(\Gamma)=\mathcal{P}(\Gamma)$, and $\mathcal{S}(\Gamma)$ contains the function $w_{0}$ such that $\left.w_{0}\right|_{\Omega_{k}} \equiv k^{-1}$. On the other hand,

$$
\sup _{j} \#\left\{g \in \Omega_{j}: w(g) \neq 0\right\}<\infty, \quad w \in \operatorname{conv} \mathcal{P}(\Gamma) .
$$

Therefore, $\left\|w_{0}-w\right\|_{\mathcal{S}}=1$ for all $w \in \operatorname{conv} \mathcal{P}(\Gamma)$.

Acknowledgements. The author is very grateful to A. Borovik, G. Brightwell, and P. Cameron for their helpful comments and encouragement. 


\section{REFERENCES}

[An] T. Ando, Majorization, doubly stochastic matrices, and comparison of eigenvalues, Linear Algebra Appl. 118 (1989), 163-248. MR0995373 (90g:15034)

[Bi1] G. Birkhoff, Three observations on linear algebra, Univ. Nac. Tucumán. Revista A 5 (1946), 147-151. (Spanish) MR0020547(8:561a)

[Bi2] L Lattice theory, Amer. Math. Soc. Colloq. Publ., vol. 25, rev. ed., Amer. Math. Soc., New York, 1948. MR0029876(10:673a)

[BR] B. R. Bapat and T. E. S. Raghavan, Nonnegative matrices and applications, Encyclopedia Math. Appl., vol. 64, Cambridge Univ. Press, Cambridge, 1997. MR1449393 (98h:15038)

[BS] J. R. Brown and R. C. Shiflett, On extreme doubly stochastic measures, Michigan Math. J. 17 (1970), 249-254. MR0265542(42:451)

[CLMST] R. M. Caron, X. Li, P. Mikusiński, H. Sherwood, and M. D. Taylor, Nonsquare "doubly stochastic" matrices, Distributions with Fixed Marginals and Related Topics (Seattle, WA, 1993), IMS Lecture Notes Monogr. Ser., vol. 28, Inst. Math. Statist., Hayward, CA, 1996, pp. 65-75. MR 1485523 (99b:15025)

[Do] R. G. Douglas, On extremal measures and subspace density, Michigan Math. J. 11 (1964), 243-246. MR0185427 (32:2894)

[Fe] D. Feldman, Doubly stochastic measures: three vignettes, Distributions with Fixed Marginals and Related Topics (Seattle, WA, 1993), IMS Lecture Notes Monogr. Ser., vol. 28, Inst. Math. Statist., Hayward, CA, 1996, pp. 84-96. MR1485525 (98k:28031)

[Gr] R. Grzaślewicz, On extreme infinite doubly stochastic matrices, Illinois J. Math. 31 (1987), 529-543. MR0909782 (88i:15046)

[Ho] A. J. Hoffman, A special class of doubly stochastic matrices, Aequationes Math. 2 (1969), 319-326. MR0263848(41:8447)

[Is] J. R. Isbell, Birkhoff's problem 111, Proc. Amer. Math. Soc. 6 (1955), 217-218. MR0068507 $(16: 893 \mathrm{f})$

[K] G. Köthe, Topological vector spaces. I, Grundlehren Math. Wiss., vol. 159, Springer-Verlag New York, Inc., New York, 1969. MR0248498 (40:1750)

[Ka] M. Katz, On the extreme points of a certain convex polytope, J. Combin. Theory 8 (1970), 417-423. MR0255582 (41:243)

[Ke] D. G. Kendall, On infinite doubly-stochastic matrices and Birkhoff's problem, J. London Math. Soc. 35 (1960), 81-84. MR0110947 (22:1815)

[KST] A. Kamiński, H. Sherwood, and M. D. Taylor, Doubly stochastic measures with mass on the graphs of two functions, Real Anal. Exchange 28 (1987/88), no 1, 253-257. MR0923729 (89f:28005)

[Le] G. Letac, Représentation des mesures de probabilité sur le produit de deux espaces dénombrables, de marges données, Illinois J. Math. 10 (1966), 497-507. MR 0200957|(34:842)

[Li] J. Lindenstrauss, A remark on extreme doubly stochastic measures, Amer. Math. Monthly $\mathbf{7 2}$ (1965), 379-382. MR0181728 (31:5955)

[LLL] J. L. Lewandowski, C. L. Liu, and J. W.-S. Liu, An algorithmic proof of a generalization of the Birkhoff-von Neumann theorem, J. Algorithms 7 (1986), no. 3, 323-330. MR0855560 (87j:15042)

[LMST] X. Li, P. Mikusiński, H. Sherwood, and M. D. Taylor, In quest of Birkhoff's theorem in higher dimensions, Distributions with Fixed Marginals and Related Topics (Seattle, WA, 1993), IMS Lecture Notes Monogr. Ser., vol. 28, Inst. Math. Statist., Hayward, CA, 1996, pp. 187-197. MR:1485531 (99b:15027)

$[\mathrm{Mu}] \quad$ H. G. Mukerjee, Supports of extremal measures with given marginals, Illinois J. Math. 29 (1985), 248-260. MR0784522 (86j:28012)

[Ro] J. V. Romanovsky, A simple proof of the Birkhoff-von Neumann theorem on bistochastic matrices, A Tribute to Ilya Bakelman (College Station, TX, 1993), Discourses Math. Appl., vol. 3, Texas A and M Univ., College Station, TX, 1994, pp. 51-53. MR.1423368 (98c:15067)

[RP] B. A. Rattray and J. E. L. Peck, Infinite stochastic matrices, Trans. Roy. Soc. Canada Sect. III (3) 49 (1955), 55-57. MR0075621 (17:778f)

[Ru] W. H. Ruckle, Sequence spaces, Res. Notes Math., vol. 49, Pitman (Advanced Publishing Program), Boston, MA-London, 1981. MR0634231 (83g:46012)

[Sa] Yu. Safarov, Birkhoff's theorem and multidimensional spectra of self-adjoint operators, J. Funct. Anal. 222 (2005), 61-97. MR2129765

[ST] H. Schreck and G. Tinhofer, A note on certain subpolytopes of the assignment polytope associated with circulant graphs, Linear Algebra Appl. 111 (1988), 125-134. MR0974049 (90e:05031) 
[Ti] G. Tinhofer, Strong tree-cographs are Birkhoff graphs, Discrete Appl. Math. 22 (1988/89), no. 3, 275-288. MR0983251 (90a:05165)

[Vi] R. A. Vitale, Parametrizing doubly stochastic measures, Distributions with Fixed Marginals and Related Topics (Seattle, WA, 1993), IMS Lecture Notes Monogr. Ser., vol. 28, Inst. Math. Statist., Hayward, CA, 1996, pp. 358-364. MR1485544 (98k:28014)

Department of Mathematics, King's College, Strand, London, United Kingdom

E-mail address: yuri.safarov@kcl.ac.uk

Received 10/MAR/2005

Translated by THE AUTHOR 